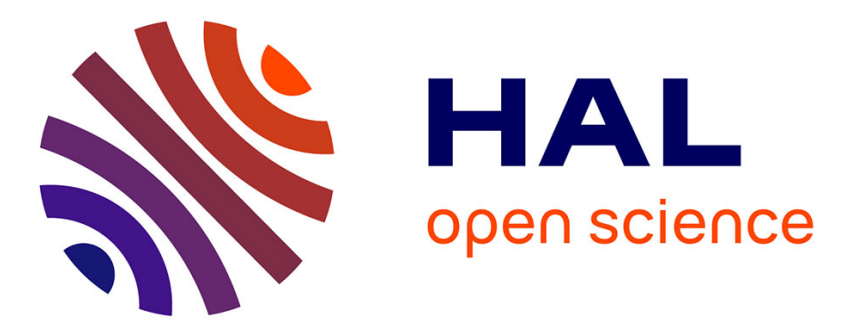

\title{
Non-linear position control of a pneumatic actuator with closed-loop stiffness and damping tuning
}

\author{
Frédéric Abry, Xavier Brun, Sylvie Sesmat, Eric Bideaux
}

\section{To cite this version:}

Frédéric Abry, Xavier Brun, Sylvie Sesmat, Eric Bideaux. Non-linear position control of a pneumatic actuator with closed-loop stiffness and damping tuning. ECC, Jul 2013, Zürich, Switzerland. pp.1089 - 1094, 10.23919/ECC.2013.6669357 . hal-00987090

\section{HAL Id: hal-00987090 https://hal.science/hal-00987090}

Submitted on 30 Apr 2019

HAL is a multi-disciplinary open access archive for the deposit and dissemination of scientific research documents, whether they are published or not. The documents may come from teaching and research institutions in France or abroad, or from public or private research centers.
L'archive ouverte pluridisciplinaire HAL, est destinée au dépôt et à la diffusion de documents scientifiques de niveau recherche, publiés ou non, émanant des établissements d'enseignement et de recherche français ou étrangers, des laboratoires publics ou privés. 


\title{
Non-linear position control of a pneumatic actuator with closed-loop stiffness and damping tuning
}

\author{
ABRY Frederic ${ }^{1}$, BRUN Xavier ${ }^{1}$, SESMAT Sylvie $^{1}$ and BIDEAUX Eric ${ }^{1}$
}

\begin{abstract}
This article proposes a new strategy for pneumatic cylinder control using an innovative control synthesis model of the system. A backstepping based control law is synthesized to take advantage of the system two degrees of freedom and a thorough tuning method of the closed-loop stiffness and damping is provided.
\end{abstract}

\section{INTRODUCTION}

Pneumatic cylinders are recognized as cheap, clean and safe actuators with a high power-to-weight ratio and dynamic response. Traditionally employed as on/off devices in order to perform very basic tasks, they most of the time do not require more than a very simple open-loop control strategy. The complexity and strong nonlinearity of their behavior and the difficulty to obtain an accurate model have for a long time prevented their use in more sophisticated applications. Yet, in the past decades, the progress of modern control theory, the enormous improvements of the microprocessor performances and the use of very efficient servovalves have made pneumatic actuators suited for most positioning operations, even those requiring high precision. The first strategies proposed [1] were mostly based upon the linearization of the model around a given steady state. Gain scheduling [2] has been introduced to improve the performances, especially when operating far from the central position. $H_{\infty}$ based control algorithms have been developed [3] to take into account errors caused by the model uncertainty and the linearization. Finally, many non-linear control strategies have been proposed: feedback linearization [4], sliding mode [5] and backstepping [6] being the most efficient techniques used so far.

One of the main particularity of a pneumatic cylinder lies in its low stiffness. Depending on the application, it can be seen as a drawback or a quality: a low stiffness means a high compliance which can be an essential characteristic, in medical applications [7] for instance, where it is required for the patient's security and comfort. On the other hand, when precision and swift disturbances rejection is needed (for example in aeronautical applications), the low stiffness decreases the actuator performances and, thus, makes it a less attractive alternative than the electro-mechanical actuators and their very high stiffness. In the past decade, strategies have been proposed to control the pneumatic stiffness of

\footnotetext{
This paper was written thanks to a research grant provided by the CNRS (Centre National de la Recherche Scientifique) and the CNES (Centre National d'Études Spatiales)

${ }^{1}$ Frederic ABRY, Xavier BRUN, Sylvie SESMAT and Eric BIDEAUX are with AMPERE, UMR 5005, Universite de Lyon, Villeurbanne, France. frederic.abryainsa-lyon. fr
}

the cylinder [8] but studies regarding the actual closed loop stiffness of the cylinder behavior have only been carried out [7] in linear positioning control. So far, the proposed multivariable non-linear control laws do not allow a precise and simple tuning of the control stiffness.

In this paper, a control algorithm is designed using an alternative state model of the cylinder and the backstepping theory in order to simultaneously control the position and pneumatic stiffness trajectories. An innovative closed loop damping and stiffness tuning is proposed making this control strategy easily implementable. Simulation results using a more detailed model are then presented to validate the chosen control law.

\section{TeChnological Context}

The proposed system consists of a symmetric in-line pneumatic cylinder supplied by two independent servovalves. The rod drives an inertial load which is submitted to heavy disturbances.

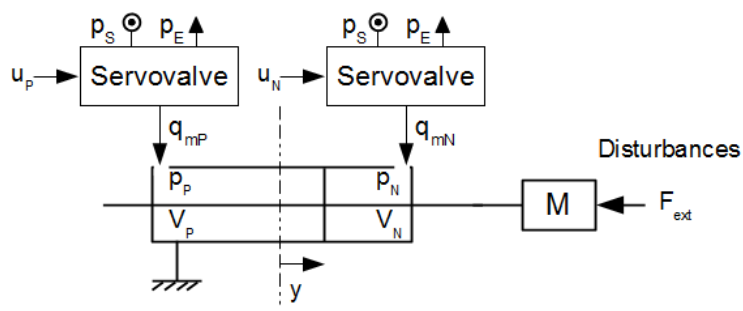

Fig. 1. Technological system under consideration

\section{Simulation Model}

\section{A. Mechanical model}

The mechanical behavior of the load is given by:

$$
M \cdot \frac{d v}{d t}=F_{p n e u}-b . v-F_{e x t}
$$

where $v$ stands for the moving mass velocity, $b$ the cylinder and load viscous friction coefficient, $F_{\text {ext }}$ the applied disturbances and $F_{\text {pneu }}$ the pneumatic force provided by the cylinder. The latter can be computed as follows: $F_{\text {pneu }}=$ $S .\left(p_{P}-p_{N}\right)$ where $p_{P}$ and $p_{N}$ stand respectively for the pressure in chambers $\mathrm{P}$ and $\mathrm{N}$ (see fig. 1) and $\mathrm{S}$ for the piston area.

\section{B. Thermodynamical model}

In order to describe the thermodynamical behavior in each chamber, the following model [9], derived from the mass and energy conservation laws, has been chosen: 
Chamber P:

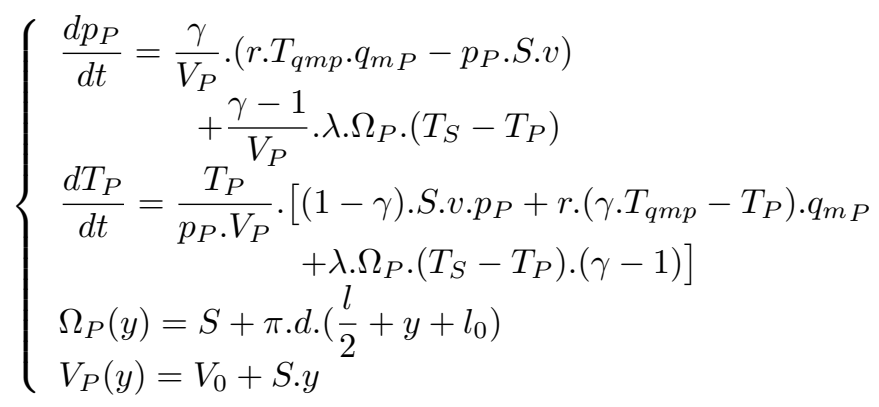

Chamber N:

$$
\left\{\begin{aligned}
\frac{d p_{N}}{d t}= & \frac{\gamma}{V_{N}} \cdot\left(r \cdot T_{q m n} \cdot q_{m N}+p_{N} \cdot S \cdot v\right) \\
& \quad+\frac{\gamma-1}{V_{N}} \cdot \lambda \cdot \Omega_{P} \cdot\left(T_{S}-T_{N}\right) \\
\frac{d T_{N}}{d t}= & \frac{T_{N}}{p_{N} \cdot V_{N}} \cdot\left[(\gamma-1) \cdot S \cdot v \cdot p_{N}+r \cdot\left(\gamma \cdot T_{q m n}-T_{N}\right) \cdot q_{m N}\right. \\
& \left.+\lambda \cdot \Omega_{N} \cdot\left(T_{S}-T_{N}\right) \cdot(\gamma-1)\right] \\
\Omega_{N}(y)= & S+\pi \cdot d \cdot\left(\frac{l}{2}-y+l_{0}\right) \\
V_{N}(y)= & V_{0}-S \cdot y
\end{aligned}\right.
$$

with $\gamma$ the ratio of specific heat, $T_{S}$ the ambient temperature, $\lambda$ the heat transfer coefficient, $r$ the ideal gas constant, $d$ the cylinder diameter, $l$ the cylinder stroke, $l_{0}$ the cylinder dead length, $q_{m P}$ and $q_{m_{N}}$ the mass flow rates defined as positive entering the chambers $\mathrm{P}$ and $\mathrm{N}$, $T_{P}$ and $T_{N}$ the respective temperatures in the $\mathrm{P}$ and $\mathrm{N}$ chambers, $V_{P}$ and $V_{N}$ the respective volumes of the $\mathrm{P}$ and $\mathrm{N}$ chambers, $V_{0}=S .\left(l_{0}+\frac{l}{2}\right)$, the cylinder half volume. $T_{q m p}$ and $T_{q m n}$ are the respective temperatures of the gaz entering or leaving the chambers $P$ and $N$ :

$T_{q m p}=\left\{\begin{array}{ll}T_{P} & \text { if } q_{m P}<0, \\ T_{S} & \text { if } q_{m P}>0\end{array} \quad T_{q m n}= \begin{cases}T_{N} & \text { if } q_{m_{N}}<0 \\ T_{S} & \text { if } q_{m_{N}}>0\end{cases}\right.$

This model takes into account the heat exchanges between the chambers and their surroundings through the varying surface areas $\Omega_{P}$ and $\Omega_{N}$.

\section{Servovalves model}

Theoretical or practical considerations show that, assuming the supply and exhaust pressures to be constant, the servovalve mass flow rate depends on both the voltage control and the chamber pressure. A simulation model [10] based on the theoretical mass flow rate of an ideal gas through a converging nozzle is chosen. The servovalve's orifice is computed as being directly proportional to the control voltage $u_{P}$ or $u_{N}$ and a second order dynamic is added to simulate the servovalve bandwidth and damping.

\section{MODEL FOR CONTROL SYNTHESIS}

Though quite accurate and representative of the actual thermodynamical behavior of both chambers, the previously proposed model is far too complex to be used to synthesize an efficient control law. Therefore, the next step is to choose a simpler representation of the cylinder.

\section{A. Simplified model}

The classical way to simplify the model is to first adopt a polytropic law and then consider the temperature variation in both chambers to be negligible. This leads to the following reduced order model [11]:

$$
\left\{\begin{array}{l}
\frac{d p_{P}}{d t}=\frac{k \cdot r \cdot T_{s}}{V_{P}} \cdot\left(q_{m P}-\frac{S}{r \cdot T_{s}} \cdot p_{P} \cdot v\right) \\
\frac{d p_{N}}{d t}=\frac{k \cdot r \cdot T_{s}}{V_{N}} \cdot\left(q_{m_{N}}+\frac{S}{r \cdot T_{s}} \cdot p_{N} \cdot v\right)
\end{array}\right.
$$

where $k$ is the polytropic coefficient chosen experimentally.

Finally, the external force is regarded as an unknown disturbance to be rejected by the control. It is thus not considered in the model for the control synthesis:

$$
\left\{\begin{array}{l}
\frac{d v}{d t}=\frac{1}{M} \cdot\left[S \cdot\left(p_{P}-p_{N}\right)-b \cdot v\right] \\
\frac{d y}{d t}=v
\end{array}\right.
$$

\section{B. Alternative input and state vectors}

The previously described model leads to the choice of the following state variables: $p_{P}, p_{N}, v$ and $y$.

Since the system have two independent inputs $q_{m P}$ and $q_{m N}$, it offers two degrees of freedom. Therefore, in addition to the position, the MIMO control strategies generally [5] propose to choose one of the chamber pressures as an output. A planned pressure trajectory can then be imposed to the system, which can offer some improvements to the energy consumption. Still, the process is somehow indirect and not entirely conclusive. In this paper, a different state vector which actually exploits the second degree of freedom and justifies the use of two independent servovalves is chosen. Therefore the pneumatic force $\left(F_{\text {pneu }}\right)$ and stiffness $\left(K_{\text {pneu }}\right)$ are controlled instead of the chambers pressures.

Thus, the first step is to choose the following state vector: $X=\left[\begin{array}{llll}y & v & F_{\text {pneu }} & K_{\text {pneu }}\end{array}\right]^{\prime}$ where [8]:

$$
K_{\text {pneu }}=\left(\frac{p_{P}}{V_{P}}+\frac{p_{N}}{V_{N}}\right) \cdot k \cdot S^{2}
$$

The model for control synthesis becomes:

$$
\left\{\begin{array}{l}
\frac{d y}{d t}=v \\
\frac{d v}{d t}=\frac{-b \cdot v+F_{\text {pneu }}}{M} \\
\frac{d F_{\text {pneu }}}{d t}=-K_{\text {pneu }} \cdot v+B_{1} \cdot q_{m} A \\
\frac{d K_{\text {pneu }}}{d t}=\frac{A_{1} \cdot K_{\text {pneu }} \cdot y \cdot v-A_{2} \cdot F_{\text {pneu }} \cdot v+B_{2} \cdot q_{m}}{V_{N} \cdot V_{P}}
\end{array}\right.
$$

where:

$$
\begin{aligned}
& A_{1}=2 \cdot S^{2} \cdot(k+1) \quad A_{2}=S^{2} \cdot k \cdot(k+1) \\
& B_{1}=S \cdot \frac{T_{s} \cdot k \cdot r}{V_{0}} \quad B_{2}=S^{2} \cdot k^{2} \cdot T_{s} \cdot r
\end{aligned}
$$




$$
\left\{\begin{array}{l}
q_{m_{H}}=q_{m_{T}}-\frac{S \cdot y}{V_{0}} \cdot q_{m_{A}} \\
{\left[\begin{array}{c}
q_{m_{A}} \\
q_{m_{T}}
\end{array}\right]=\Lambda(y) \cdot\left[\begin{array}{l}
q_{m_{P}} \\
q_{m_{N}}
\end{array}\right]}
\end{array}\right.
$$

with the following transform matrix:

$$
\Lambda(y)=V_{0} \cdot\left[\begin{array}{cc}
-\frac{1}{V_{P}} & \frac{1}{V_{N}} \\
\frac{1}{V_{P}} & \frac{1}{V_{N}}
\end{array}\right]
$$

Thus, the system shows two independent virtual inputs $q_{m_{A}}$ and $q_{m_{T}}$ which control two different behaviors of the cylinder. $q_{m_{A}}$ is the active mass flow rate which actually generates a pressure difference between the two chambers and therefore an effort which ultimately can put the carried mass into motion. On the contrary, $q_{m_{T}}$, the pressurization mass flow rate, does not induce a pneuamtic force and can only lead to a symmetric pressurization of the chambers. It has to be noted that the pneumatic stiffness $K_{\text {pneu }}$ dynamics depend on both $q_{m_{A}}$ and $q_{m_{T}}$.

This rewriting of the model, can somehow be compared to the Park Transform [12] in electric motor control (which involves distinguishing the two current components respectively generating the flux and the torque).

Finally, the proposed equations of state show a strict feedback form which is particularly suited for control synthesis, especially using the backstepping theory.

\section{BACKSTEPPING CONTROL SYNTHESIS}

\section{A. Mass flow rate generation}

In the previously proposed model, the mass flow rates have been considered as the input vector. Obviously, those are not directly controllable, the actual outputs of the control algorithm have to be the servovalves voltage inputs.

Mostly two distinct strategies are proposed in the literature, the first one consists in using a theoretical model like the one previously cited in III-C. Mass flow rate parameters are then estimated using the supplier data sheet or by experimental tests. The second technique consists in a thorough experimental evaluation of the servovalve mass flow rate for different voltage controls and chamber pressures [13]. From the results is derived a three dimensional table giving the flow rate for each couple pressure - control voltage. In order to reduce heavy computation load, a polynomial function is derived from the table. The results have proven to be fairly precise but since computational capacities have significantly increased since this method has been proposed, there is no need for this approximation anymore. Therefore, in this paper, an experimental table is used to directly derive the control voltage by a simple weighted mean of the surrounding values. Servovalves dynamics are neglected since they are supposed to be very fast compared to the pressure dynamics.

\section{B. Control law synthesis - position trajectory tracking}

The first part of the control law is defined to ensure the tracking of a trajectory defined by $j_{d}$ the desired jerk and its successive integrals $a_{d}, v_{d}$ and $y_{d}$. $z_{1}=y-y_{d}$ is defined as the position error, its derivative can be computed as: $\dot{z}_{1}=v-v_{d}$

At this point, $v$ is viewed as a virtual input and has to be chosen to cancel and stabilize the error $z_{1}$ :

$$
v=v_{d}-C_{1} \cdot z_{1}
$$

with $C_{1}$ a strictly positive constant. To assess the global stability of the subsystem, the following Lyapunov function: $V_{1}=\frac{z_{1}^{2}}{2} \geq 0$ is chosen. Its derivative can be computed:

$$
\dot{V}_{1}=-C_{1} \cdot z_{1}^{2} \leq 0
$$

Its negativity ensures the stability of the subsystem, the next error variable is defined as: $z_{2}=v-v_{d}+C_{1} . z_{1}$. It leads to:

$$
\begin{gathered}
\dot{z_{1}}=z_{2}-C_{1} \cdot z_{1} \\
\dot{z_{2}}=\frac{F_{\text {pneu }}-b \cdot v}{M}-a_{d}+C_{1} \cdot z_{2}-C_{1}^{2} \cdot z_{1}
\end{gathered}
$$

A new Lyapunov candidate $V_{2}=V_{1}+\frac{z_{2}^{2}}{2} \geq 0$ and the following pneumatic effort control are defined:

$F_{\text {pneu }_{d}}=M \cdot\left(a_{d}-z_{2} \cdot\left(C_{1}+C_{2}\right)+z_{1} \cdot\left(C_{1}^{2}-1\right)\right)+b \cdot v$

with $C_{2}$ a strictly positive constant. If $F_{\text {pneu }}=F_{\text {pneud }}$ is ensured, then the error derivative can be computed as $\dot{z}_{2}=$ $-z_{1}-C_{2} . z_{2}$ which eventually leads to $\dot{V}_{2}=-C_{1} . z_{1}^{2}-C_{2} . z_{2}^{2}$. The negativity of the derivative ensures the subsystem global stability. The next error variable is then introduced:

$$
z_{3}=F_{\text {pneu }}-F_{\text {pneu }}
$$

It leads to:

$$
\begin{gathered}
\dot{z}_{2}=\frac{z_{3}}{M}-z_{1}-C_{2} \cdot z_{2} \\
\dot{z_{3}}=B_{1} \cdot q_{m A}-K_{\text {pneu }} \cdot v-M \cdot j_{d}-\frac{b \cdot\left(F_{\text {pneu }}-b \cdot v\right)}{M} \\
+M .\left(C_{1}^{3}-2 \cdot C_{1}-C_{2}\right) \cdot z_{1}+\left(C_{1}+C_{2}\right) \cdot z_{3} \\
+M .\left(1-C_{1}^{2}-C_{2}^{2}-C_{1} \cdot C_{2}\right) \cdot z_{2}
\end{gathered}
$$

A third Lyapunov function can be defined as:

$$
V_{3}=V_{2}+\frac{z_{3}^{2}}{2}
$$

If the following active mass flow rate control is chosen:

$$
q_{m_{A}}=f_{0}+f_{1} \cdot z_{1}+f_{2} \cdot z_{2}+f_{3} \cdot z_{3}
$$

where:

$$
\begin{aligned}
& f_{0}=\frac{M^{2} \cdot j_{d}+M \cdot K_{\text {pneu }} \cdot v-v \cdot b^{2}+F_{\text {pneu }} \cdot b}{M \cdot B_{1}} \\
& f_{1}=-\frac{M \cdot\left(C_{1}^{3}-2 \cdot C_{1}-C_{2}\right)}{B_{1}} \\
& f_{2}=\frac{M^{2} \cdot\left(C_{1}^{2}+C_{1} \cdot C_{2}+C_{2}^{2}-1\right)-1}{M \cdot B_{1}} \\
& f_{3}=-\frac{C_{1}+C_{2}+C_{3}}{B_{1}}
\end{aligned}
$$


with $C_{3}$ a strictly positive constant, then $\dot{V}_{3}=-C_{1} \cdot z_{1}^{2}-$ $C_{2} . z_{2}^{2}-C_{3} . z_{3}^{2} \leq 0$ and the error will asymptoticly converge to zero.

In this first part, a virtual mass flow-rate $q_{m}$ which ensures that the cylinder will follow the desired trajectory has been computed. At this point, no choice has been made regarding the $q_{m T}$ control.

\section{Pneumatic stiffness trajectory tracking}

As previously mentioned the position trajectory tracking only uses one of the system two degrees of freedom. Consequently, a control law can be synthesized to ensure the tracking of a given pneumatic stiffness trajectory.

A new error variable can therefore be defined: $z_{4}=K_{\text {pneu }}-$ $K_{\text {pneu }}$. Its derivative can be computed as follows:

$\dot{z}_{4}=\frac{A_{1} \cdot K_{\text {pneu }} \cdot v \cdot y-A_{2} \cdot F_{\text {pneu }} \cdot v+B_{2} \cdot q_{m H}}{V_{N} \cdot V_{P}}-\dot{K}_{\text {pneu }_{d}}$

A final Lyapunov function has to be defined: $V_{4}=V_{3}+$ $\frac{z_{4}^{2}}{2} \geq 0$ and the following pressurization mass flow rate control is chosen:

$$
\begin{aligned}
q_{m_{H}}= & \frac{1}{B_{2}} \cdot\left[A_{2} \cdot F_{\text {pneu }} \cdot v+V_{N} \cdot V_{P} \cdot\left(\dot{K}_{\text {pneu }_{d}}-C_{4} \cdot z_{4}\right)\right. \\
& \left.-A_{1} \cdot K_{\text {pneu }} \cdot v \cdot y\right]
\end{aligned}
$$

with $C_{4}$ a strictly positive constant. It leads to: $\dot{V}_{4}=$ $-C_{1} . z_{1}^{2}-C_{2} . z_{2}^{2}-C_{3} . z_{3}^{2}-C_{4} . z_{4}^{2} \leq 0$ which means that the cylinder pneumatic stiffness will track the trajectory defined by $\dot{K}_{\text {pneu }_{d}}$ and its integral $K_{\text {pneu }}$.

The pneumatic stiffness trajectory tracking leads to a virtual mass flow rate $q_{m_{H}}$ control which depends on both $q_{m A}$ and $q_{m_{T}}$, the first one being computed to track the position trajectory. From (7), the actual mass flow rate controls can be calculated through the following relationships:

$$
\begin{array}{r}
q_{m_{T}}=q_{m_{H}}+\frac{S \cdot y}{V_{0}} \cdot q_{m_{A}} \\
{\left[\begin{array}{l}
q_{m_{P}} \\
q_{m_{N}}
\end{array}\right]=\Lambda^{-1}(y) \cdot\left[\begin{array}{l}
q_{m_{A}} \\
q_{m_{T}}
\end{array}\right]}
\end{array}
$$

The whole control law (fig. 2) requires the measurement of both pressures $p_{P}$ and $p_{N}$ as well as the piston position $y$ and velocity $v$.

\section{PARAMETERS TUNING}

The tuning of non-linear control strategy is always a complex task since the control parameters seldom show an obvious physical meaning. It is usually done by trial and error which is time consuming, complex and inaccurate, often preventing the algorithm from being used in industrial applications. In this section is proposed a new simple yet very efficient way to choose the parameters by defining the cylinder behavior using linear concepts.

\section{A. Definition of the closed-loop stiffness}

The closed-loop stiffness is defined as $K_{c l}=-\frac{d \sum F}{d z_{1}}$, where $\sum F$ stands for the sum of the forces applied on the piston according to the control synthesis model (6) and $z_{1}$ the previously defined position error.

$$
\begin{aligned}
& K_{c l}=-\frac{d F_{\text {pneu }}}{d z_{1}}-\frac{d(-b \cdot v)}{d z_{1}} \\
& =-M \cdot \frac{d\left(-z_{2} \cdot\left(C_{1}+C_{2}\right)+z_{1} \cdot\left(C_{1}^{2}-1\right)\right)}{d z_{1}}
\end{aligned}
$$

and, since $z_{2}=v-v_{d}+C_{1} \cdot z_{1}$ :

$$
K_{c l}=-M \cdot \frac{d\left(-C_{1} \cdot z_{1} \cdot\left(C_{1}+C_{2}\right)+z_{1} \cdot\left(C_{1}^{2}-1\right)\right)}{d z_{1}}
$$

And finally:

$$
K_{c l}=M \cdot\left(C_{1} \cdot C_{2}+1\right)
$$

It has to be noted that $K_{c l}$ and $K_{\text {pneu }}$, while both having the dimension of a stiffness, represent two independent concepts: $K_{\text {pneu }}$ is the pneumatic stiffness, a physical intrinsic property of the cylinder which is chosen as a state variable to be controlled while $K_{c l}$ is the closed-loop stiffness, a parameter of the behavior of the system controlled by the previously proposed algorithm, it does not depends on the position of the piston.

\section{B. Definition of the closed-loop damping}

Likewise, the closed-loop damping can be defined as $B_{c l}=-\frac{d \sum F}{d \bar{v}}$ with $\bar{v}=v-v_{d}$.

$$
B_{c l}=-\left(\frac{d F_{\text {pneu }}}{d \bar{v}}-\frac{b . v}{d \bar{v}}\right)
$$

Since $v=\bar{v}+v_{d}, \frac{d v}{d \bar{v}}=\frac{d \bar{v}}{d \bar{v}}+\frac{d\left(v_{d}\right)}{d \bar{v}}=1$

$$
\begin{aligned}
& B_{c l}=-\frac{d\left(M \cdot\left(a_{d}+z_{1} \cdot\left(C_{1}^{2}-1\right)-z_{2} \cdot\left(C_{1}+C_{2}\right)\right)\right.}{d \bar{v}} \\
& =-\frac{d\left(-M \cdot z_{2} \cdot\left(C_{1}+C_{2}\right)\right)}{d \bar{v}}
\end{aligned}
$$

And finally:

$$
B_{c l}=M \cdot\left(C_{1}+C_{2}\right)
$$

\section{Computation of the control law parameters}

For a given closed-loop stiffness $K_{c l}$ and closed-loop damping $B_{c l}$, the corresponding $C_{1}$ and $C_{2}$ parameters have to be computed. From (26) the following can be derived:

$$
C_{2}=\frac{K_{c l}-M}{C_{1} \cdot M}
$$

The global stability of the system requires $C_{2}>0$ which leads to $K_{c l}>M$. From (29) and (30) can be derived:

$$
M . C_{1}^{2}-B_{c l} . C_{1}+K_{c l}-M=0
$$


Solutions of this second order equation have to be real and positive, therefore: $B_{c l} \geq 2 \sqrt{M .\left(K_{c l}-M\right)}$ There is only one practical solution because $C_{1}$ and $C_{2}$ values are interchangeable:

$$
\left\{\begin{aligned}
C_{1} & =\frac{B_{c l}+\sqrt{B_{c l}^{2}-4 \cdot M \cdot\left(K_{c l}-M\right)}}{2 \cdot M} \\
C_{2} & =\frac{B_{c l}-\sqrt{B_{c l}^{2}-4 \cdot M \cdot\left(K_{c l}-M\right)}}{2 \cdot M}
\end{aligned}\right.
$$

The two conditions:

$$
\left\{\begin{array}{l}
B_{c l} \geq 2 \sqrt{M \cdot\left(K_{c l}-M\right)} \\
K_{c l}>M
\end{array}\right.
$$

ensure the global stability of the system.

\section{Closed-loop regulation behavior}

According to (13), (26) and (29), the pneumatic force control provided by the backstepping based algorithm can be expressed as follows:

$$
F_{\text {pneu }_{d}}=-K_{c l} \cdot z_{1}-B_{c l} \cdot \bar{v}+M . a_{d}+b . v
$$

The closed loop behavior of the cylinder in regulation when submitted to an unknown external force $F_{\text {ext }}$ can be described as:

$$
M \cdot \frac{d v}{d t}=\sum F=F_{\text {pneu }}-b \cdot v-F_{\text {ext }}
$$

For a constant set-position, that is for $v_{d}=a_{d}=j_{d}=0$, the approximation $F_{\text {pneu }}=F_{\text {pneu }}$ is made:

$$
M \cdot \frac{d v}{d t}=-K_{c l} \cdot z_{1}-B_{c l} \cdot \bar{v}-F_{e x t}
$$

Since $v_{d}=0, \bar{v}=v, v=\dot{z_{1}}$.

$$
M . \ddot{z}_{1}=-K_{c l} . z_{1}-B_{c l} . \dot{z}_{1}-F_{e x t}
$$

This study neglects the error $z_{3}$ defined by (14) and therefore has absolutely no value as a stability proof but merely provides information about the algorithm tuning. The piston response to an external disturbance in the Laplace domain can be expressed as follows:

$$
H_{r e g}=\frac{z_{1}}{F_{\text {ext }}}=-\frac{1}{M . s^{2}+B_{c l} . s+K_{c l}}
$$

This transfer function corresponds to a classical spring-mass system with friction. The gain $G$, natural frequency $\omega_{n}$ and damping ratio $\xi$ of the second order transfer function can be computed:

$$
G=-\frac{1}{K_{c l}} \quad ; \quad \omega_{n}=\sqrt{\frac{K_{c l}}{M}} \quad ; \quad \xi=\frac{B_{c l}}{2 \cdot \sqrt{K_{c l} \cdot M}}
$$

Which leads to the following tuning rules:

- the condition for a non-oscillatory response of the piston to an external force is: $B_{c l} \geq 2 \cdot \sqrt{K_{c l} \cdot M}$

- the steady-state error can be computed as $\Delta_{y}=-\frac{F_{e x t}}{K_{c l}}$ therefore increasing the system closed-loop stiffness will reduce the static error
- the damping ratio is proportional to $B_{c l}$. This implies that, in the non-oscillatory response case, the convergence time will be increased by a high closed-loop damping, which traduces the control ability to slow down the piston without altering the steady-state position.

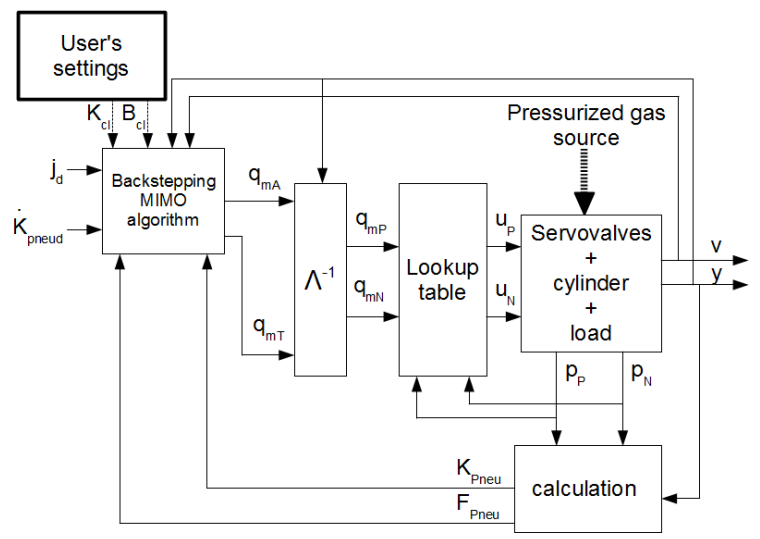

Fig. 2. Complete principle of the control strategy

\section{E. Remaining parameters}

A thorough physical analysis of the $C_{3}$ and $C_{4}$ parameters cannot be provided in this paper, it has to be the object of a dedicated study. Still, a simple observation of (18) and (21) shows that they respectively affect the derivatives $\frac{d q_{m_{A}}}{d z_{3}}$ and $\frac{d q_{m_{H}}}{d z_{4}}$. In others words, the settings of $C_{3}$ and $C_{4}$ will respectively define the levels of the pneumatic effort and pneumatic stiffness error feedback.

\section{F. Simulation results}

To illustrate those results, a simulation is performed using the model proposed in section III. The piston is controlled to move to a given position and stay still. An external force step (non represented for the sake of brevity) of $20 \%$ of the cylinder maximum pneumatic force is applied at $\mathrm{t}=$ $2.5 \mathrm{~s}$ until $\mathrm{t}=7.5 \mathrm{~s}$. A constant pneumatic stiffness trajectory is chosen and the piston behavior is simulated for three different tunings (table. I). Position and pneumatic stiffness responses can be seen in fig. 3 and fig. 4.

\begin{tabular}{|l|c|c|c|}
\hline Trial & $\# 1$ & $\# 2$ & $\# 3$ \\
\hline$K_{c l}\left[N . m^{-1}\right]$ & $1.10^{5}$ & $1.10^{5}$ & $5.10^{5}$ \\
\hline$B_{c l}\left[N . s . m^{-1}\right]$ & $2.5 .10^{3}$ & $7.5 .10^{3}$ & $5.5 .10^{3}$ \\
\hline$\xi$ & 1.02 & 3.06 & 1.00 \\
\hline$\omega_{n}\left[\right.$ rad.s $\left.{ }^{-1}\right]$ & 8.17 & 8.17 & 18.26 \\
\hline
\end{tabular}

TABLE I

The initial displacement shows the expected position trajectory tracking in the three cases but the response to the external disturbance differs according to the chosen tuning. The first trial is performed with a low closed-loop stiffness and the corresponding minimal closed-loop damping. Convergence is quick and with no oscillations on the position. The second trial is made with the same closed-loop stiffness but a higher closed-loop damping, the piston displacement is slowed-down and convergence time is greatly increased in accordance with the higher value of the damping ratio $\xi$ but 
the position static error is almost unchanged (the small difference being the result of the pneumatic force tracking error neglected in section VI). Finally, the last trial shows the result of a five times higher closed-loop stiffness: the static error is proportionally reduced. Results are summarized in table II. Expected static errors are computed as $\Delta_{y}=-\frac{F_{e x t}}{K_{c l}}$. Expected settling times (time necessary for the piston to reach and remain within $5 \%$ of its steady state position) are computed using a classical algebraic approximation for second order systems.

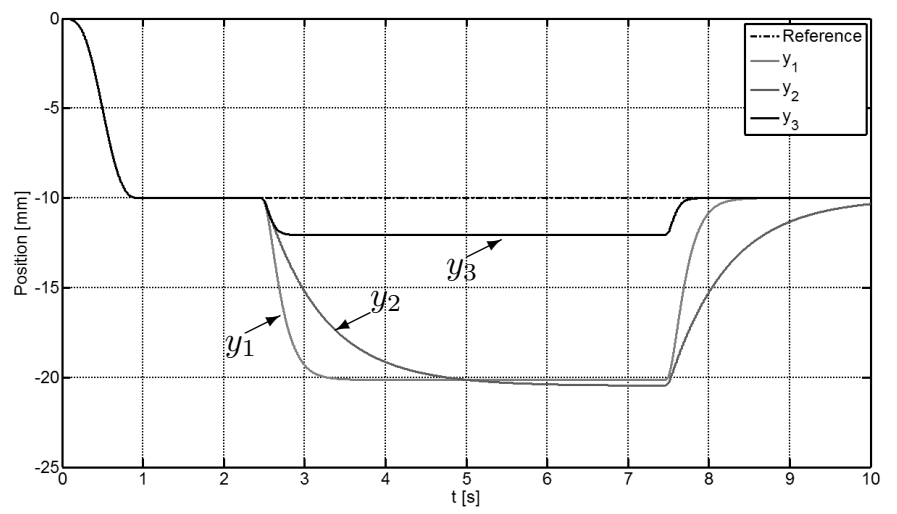

Fig. 3. Position response of the piston for various tunings

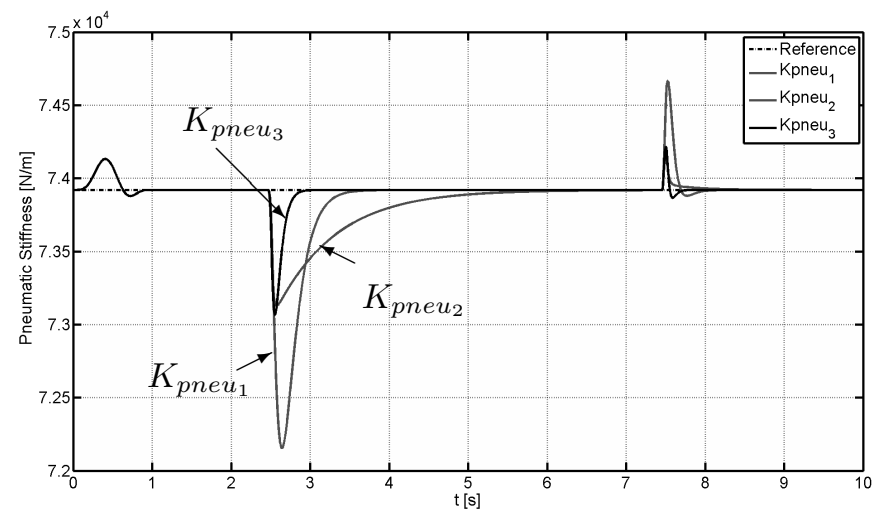

Fig. 4. Pneumatic stiffness response of the cylinder for various tunings

\begin{tabular}{|l|c|c|c|}
\hline Trial & $\# 1$ & $\# 2$ & $\# 3$ \\
\hline Expected static error [mm] & 10 & 10 & 2 \\
\hline Simulated static error [mm] & 10.13 & 10.45 & 2.07 \\
\hline Expected settling time [s] & 0.62 & 2.28 & 0.28 \\
\hline Simulated settling time [s] & 0.579 & 2.167 & 0.241 \\
\hline
\end{tabular}

TABLE II

The provided simulations have showed the efficiency of the control strategy and validated the tuning method. The simulated cylinder non-linear behavior has proved to be very close to the provided linear approximation and the closed-loop stiffness and damping parameters are precisely respected.

\section{CONCLUSION}

In this paper a transformation of the traditional pneumatic cylinder control synthesis model has been introduced. It makes easier and more intuitive the synthesizing of nonlinear control laws taking advantage of the system two degrees of freedom.
A study of the backstepping based algorithm has led to the development of an innovative tuning strategy which allows the computation of the control parameters using linear concepts such as stiffness and damping. This development simplifies the control law implementation and therefore makes non-linear position control of pneumatic cylinder more adapted to engineering problems.

The next step of this study will provide experimental results: a test-bench using an electric linear motor as a disturbance generator (currently in the final stage of assembly) will offer the possibility to assess the controlled pneumatic cylinder response when submitted to swift perturbations.

The remaining parameters, which are not computed by the proposed strategy, have to be studied in the same way in order to offer a complete solution for the algorithm tuning.

A study also needs to be conducted on the pneumatic stiffness trajectory and how it has to be chosen depending on the objectives of the control: strong disturbances rejection or fast response to a displacement set-point value.

Finally, a theoretical analysis could demonstrate the system global stability when submitted to a bounded disturbance.

\section{REFERENCES}

[1] J.L. Shearer. Study of pneumatic processes in the continuous control of motion with compressed air, volume 78, pages 233-249. Trans. Am. Soc. Mech. Engrs, 1956.

[2] X. Brun, M. Belgharbi, S. Sesmat, D. Thomasset, and S. Scavarda. Control of an electropneumatic actuator: comparison between some linear and non-linear control laws. In Proceedings of the Institution of Mechanical Engineers, Part I: Journal of Systems and Control Engineering, volume 213, pages 387-406. Sage Publications, 1999.

[3] T. Kimura, S. Hara, and T. Tomisaka. $h_{\infty}$ control with minor feedback for a pneumatic actuator system. In Decision and Control, 1996., Proceedings of the 35th IEEE, volume 3, pages 2365-2370, dec 1996.

[4] T. Fujita T. Kimura, S. Hara and T. Kagawa. Feedback linearization for pneumatic actuator systems with static friction. Control Engineering Practice, 5(10):1385-1394, 1997.

[5] M. Smaoui, X. Brun, and D. Thomasset. A combined first and second order sliding mode approach for position and pressure control of an electropneumatic system. In American Control Conference, 2005. Proceedings of the 2005, pages 3007-3012 vol. 5, june 2005.

[6] M. Smaoui, X. Brun, and D. Thomasset. A study on tracking position control of an electropneumatic system using backstepping design. Control Engineering Practice, 14(8):923-933, 2006.

[7] R. Moreau, M.T. Pham, X. Brun, T. Redarce, and O. Dupuis. Simulation of an instrumental childbirth for the training of the forceps extraction: control algorithm and evaluation. IEEE Transactions on Information Technology in Biomedicine, 15(3):364 - 372, January 2011.

[8] X. Shen and M. Goldfarb. Simultaneous force and stiffness control of a pneumatic actuator. Journal of Dynamic Systems, Measurement, and Control, 129(4):425-434, 2007.

[9] H.S. Jebar. Design of pneumatic actuator systems. PhD thesis, University. Of Nottingham, 1977.

[10] D. Mc Cloy. Discharge characteristics of servo valve orifices. In Fluid Power International conference, number 6, pages 43-503, 1968.

[11] A. Chitty and T.H. Lambert. Modelling a loaded two-way pneumatic actuator. Journal of Dynamic Systems Measurements and Control, 9(1):19-25, 1976

[12] W. Leonhard. Control of electrical Drives. Berlin Springer-Verlag, corrected 2nd printing edition, 1990.

[13] M. Belgharbi, S. Sesmat, S. Scavarda, and D. Thomasset. Analytical model of the flow stage of a pneumatic servo-distributor for simulation and nonlinear control. The sixth Scandinavian international conference on fluid power, pages p847-860, May 26-28 1999. 